\title{
Anti-TIGIT differentially affects sepsis survival in immunologically experienced versus previously naive hosts
}

\author{
Yini Sun, ${ }^{1,2,3}$ Jerome C. Anyalebechi, ${ }^{1,3}$ He Sun, ${ }^{1,4,5}$ Tetsuya Yumoto, ${ }^{1,3}$ Ming Xue, ${ }^{1,3,6}$ Danya Liu, ${ }^{1,5}$ \\ Zhe Liang, ${ }^{1,3}$ Craig M. Coopersmith, ${ }^{1,3}$ and Mandy L. Ford ${ }^{1,5}$ \\ 'Department of Surgery, Emory University School of Medicine, Atlanta, Georgia, USA. ²Department of Critical Care \\ Medicine, The First Affiliated Hospital of China Medical University, China Medical University, Shenyang, China. ${ }^{3}$ Emory \\ Critical Care Center, Emory University School of Medicine, Atlanta, Georgia, USA. ${ }^{4}$ Department of Hepatobiliary Surgery \\ and Transplantation, The First Affiliated Hospital of China Medical University, China Medical University, Shenyang, China. \\ ${ }^{5}$ Emory Transplant Center, Emory University School of Medicine, Atlanta, Georgia, USA. ${ }^{6}$ Department of Critical Care \\ Medicine, Zhongda Hospital, School of Medicine, Southeast University, Nanjing, China.
}

Mounting evidence suggests that the balance of T cell costimulatory and coinhibitory signals contributes to mortality during sepsis. Here, we identified a critical role of the coinhibitory molecule T cell Ig and ITIM domain (TIGIT) in regulating sepsis mortality. Because TIGIT is significantly upregulated on memory T cells, we developed a "memory mouse" model to study the role of TIGIT during sepsis in a more physiologically relevant context. Mice received sequential pathogen exposure and developed memory $\mathrm{T}$ cell frequencies, similar to those observed in adult humans, and were then subjected to sepsis induction via cecal ligation and puncture. Our results show that targeting the TIGIT pathway during sepsis is fundamentally different in previously naive versus memory mice, in that $\alpha$ TIGIT Ab had no effect on survival in previously naive septic mice but sharply worsened survival in memory septic mice. Mechanistically, $\alpha$ TICIT increased apoptosis of memory T cells, decreased T cell function, and downregulated the costimulatory receptor DNAM on memory CD8 ${ }^{+} \mathrm{T}$ cells in memory septic mice, but not in previously naive septic mice. Additionally, $\alpha$ TICIT diminished Helios expression in Tregs in memory but not previously naive septic mice. These data highlight fundamental differences in the pathophysiological impact of targeting TIGIT in immunologically experienced versus previously naive hosts during sepsis.

Authorship note: CMC and MLF contributed equally to this work.

Conflict of interest: The authors have declared that no conflict of interest exists.

Copyright: (ㄷ) 2021, Sun et al. This is an open access article published under the terms of the Creative Commons Attribution 4.0 International License.

Submitted: June 10, 2020

Accepted: January 20, 2021

Published: March 8, 202

Reference information: /CI Insight. 2021;6(5):e141245.

https://doi.org/10.1172/jci. insight.141245.

\section{Introduction}

Sepsis is a life-threatening organ dysfunction that results from a dysregulated host response to infection (1). It is the most common cause of death in the intensive care unit (ICU), resulting in $20 \%$ of ICU deaths worldwide (2). Although sepsis has been historically considered to be the result of an overwhelming inflammatory response, emerging evidence has demonstrated that disruption in the balance of costimulatory versus coinhibitory signaling contributes to increased morbidity and mortality during sepsis (3).

Accumulating evidence also suggests that apoptosis and dysfunction of $\mathrm{T}$ cells contributes to sepsis-induced immunosuppression (4). Additionally, analysis of samples from patients who died of sepsis revealed decreased $\mathrm{T}$ cell numbers, decreased $\mathrm{T}$ cell cytokine production, and increased $\mathrm{T}$ cell coinhibitory receptor expression (5). Specifically, T cell coinhibitory receptors PD-1, CTLA-4, and 2B4 have been shown to be highly expressed in septic mice and humans, and are being targeted clinically to improve antiseptic responses (6). Anti-PD-1/PD-L1 mAbs have been reported to improve the survival in experimental models of sepsis, and recent clinical trials are beginning to report positive outcomes $(7,8)$. Blockade of CTLA-4 also resulted in increased survival of the murine cecal ligation and puncture (CLP) model (9). Moreover, we recently demonstrated that blockade of the coinhibitory molecule 2B4 resulted in increased CLP survival in both previously healthy septic animals and in septic mice with preexisting malignancy $(10,11)$. Taken together, these studies demonstrate the potential therapeutic utility of checkpoint blockade in sepsis.

$\mathrm{T}$ cell Ig and ITIM domain (TIGIT) is a novel coinhibitory molecule that is expressed on activated human $\mathrm{T}$ cells and contains an Ig variable domain, a transmembrane domain, and an ITIM (12). 
TIGIT is mainly expressed on memory T cells, Tregs, as well as NK cells, and is upregulated on naive T cells upon activation. Similar to the CTLA-4/CD28 pathway, TIGIT competes with the costimulatory receptor CD226 (also known as DNAM) for the same set of ligands (CD155 and CD112) and mediates immune suppression in tumors and chronic infections (13-15). Our previous study showed that TIGIT expression on $\mathrm{T}$ and NK cells was upregulated in the mouse model of sepsis (our unpublished observations).

It is also well known that TIGIT expression is significantly altered on memory $\mathrm{T}$ cells compared with naive $\mathrm{T}$ cells in humans and mice (12). However, laboratory mice possess fewer memory $\mathrm{T}$ cells than adult humans or feral mice (16). Therefore, to better mimic the immune features of adults and explore the role of TIGIT in sepsis, we developed a "memory mouse" model, in which mice receive sequential pathogen exposure and possess frequencies of memory $\mathrm{T}$ cells similar to those observed in adult humans, followed by sepsis induction via CLP (17). We then interrogated the role of TIGIT signaling on memory T cells and explored the differential effects of $\alpha$ TIGIT on memory versus previously naive mice during sepsis. Results indicated that whereas $\alpha$ TIGIT had no effect on sepsis survival in previously naive septic mice, it resulted in significantly decreased survival in memory septic mice. Moreover, $\alpha$ TIGIT mAb exhibited markedly different effects on $\mathrm{T}$ cell apoptosis, cytokine effector function, and Treg stability in previously naive versus immunologically experienced hosts during sepsis.

\section{Results}

TIGIT expression on both $C D 4^{+}$and $C D 8^{+} T$ cells is upregulated in memory mice relative to naive mice. Multiple studies have shown that in addition to Tregs, TIGIT is primarily expressed on memory $\mathrm{T}$ cells and is absent on naive T cells $(12,14)$. To study TIGIT biology in a more physiologically relevant context, we developed a memory mice model via sequential infection of naive B6 mice with Listeria monocytogenes followed by lymphocytic choriomeningitis virus (LCMV) (Supplemental Figure 1A; supplemental material available online with this article; https://doi.org/10.1172/jci.insight.141245DS1). At 56 days after first infection, these antigen-experienced memory mice possessed approximately $23 \%$ memory $\mathrm{CD} 44^{\mathrm{hi}} \mathrm{CD} 4^{+} \mathrm{T}$ cells and $66 \% \mathrm{CD}_{4}{ }^{\mathrm{hi}} \mathrm{CD}^{+} \mathrm{T}$ cells in the blood, which is similar to the frequencies observed in adult humans (Supplemental Figure 1B) (16). In contrast, $\mathrm{CD} 4^{+}$and $\mathrm{CD}^{+} \mathrm{T}$ cell compartments of age-matched naive mice comprised only $15 \%$ and $19 \%$ memory T cells, respectively (Supplemental Figure 1, C and D). Additionally, the absolute numbers of memory $\mathrm{CD}^{+}$and $\mathrm{CD} 8^{+} \mathrm{T}$ cells in memory mice were also dramatically higher than those in naive mice $\left(\mathrm{CD} 44^{\mathrm{hi}} \mathrm{CD} 4^{+}, P=0.0007\right.$, Supplemental Figure $1 \mathrm{E} ; \mathrm{CD} 44^{\mathrm{hi}} \mathrm{CD} 8^{+}, P=0.0007$, Supplemental Figure 1F).

To determine TIGIT expression on T cells, splenocytes from naive and memory mice were analyzed by flow cytometry (Supplemental Figure 1G). TIGIT expression was significantly increased on bulk CD4 ${ }^{+}$and $\mathrm{CD}^{+} \mathrm{T}$ cells in memory mice compared with naive mice (CD4: $5.4 \% \pm 0.3 \%$ vs. $7.4 \% \pm 0.5 \%, P=0.0037$, Supplemental Figure $1 \mathrm{H}$; CD8: $1.7 \% \pm 0.3 \%$ vs. $2.9 \% \pm 0.3 \%, P=0.04$, Supplemental Figure $1 \mathrm{I})$. Compared with naive mice, the percentage of TIGIT on CD $44^{\text {hi }} \mathrm{CD} 8^{+} \mathrm{T}$ cells was still increased in memory mice ( $3.2 \pm 0.5$ vs. $5.1 \pm 0.6, P=0.03)$, although there was no difference in TIGIT expression on $\mathrm{CD} 44^{\text {hi }} C D 4^{+} \mathrm{T}$ cells between memory and naive mice (Supplemental Figure 1, J and K).

TIGIT expression is higher on $C D 8^{+} T$ cells in memory versus previously naive mice during sepsis. To further determine TIGIT expression on $\mathrm{T}$ cells during sepsis, splenocytes from previously naive and memory mice that had undergone CLP surgery were analyzed by flow cytometry at 48 hours after CLP (Figure 1A). TIGIT expression on both bulk CD4 ${ }^{+}$and $\mathrm{CD} 44^{\mathrm{hi}} \mathrm{CD} 4^{+} \mathrm{T}$ cells in memory septic mice did not differ from that in previously naive septic mice (Figure 1, B, C, and E). In contrast, TIGIT expression was increased on bulk $\mathrm{CD}^{+} \mathrm{T}$ cells in memory mice compared with previously naive mice during sepsis $(2.9 \% \pm 0.6 \%$ vs. $5.3 \% \pm$ $0.6 \%, P=0.028$, Figure $1, \mathrm{~B}$ and $\mathrm{D})$. Further, TIGIT expression on CD $44^{\mathrm{hi}} \mathrm{CD} 8^{+} \mathrm{T}$ cells was significantly enhanced in memory mice relative to previously naive mice at 48 hours after CLP ( $4.6 \% \pm 0.7 \%$ vs. $15.9 \%$ $\pm 2.1 \%, P=0.0002$, Figure $1, \mathrm{~B}$ and $\mathrm{F}$ ).

$\alpha T I G I T$ Ab worsens sepsis survival in memory but not previously naive hosts. First, we assessed the impact of blocking TIGIT signaling in previously naive mice by interrogating 7-day survival after CLP in the presence of treatment with the $\alpha$ TIGIT Ab or isotype control Ab (Figure 2A). We confirmed that this clone, previously reported to be blocking (18), was in fact blocking and not depleting at the concentrations used because in vivo administration of the $\mathrm{Ab}$ inhibited ex vivo staining with a fluorescently labeled anti-TIGIT, but without any change in $\mathrm{CD}^{+}$or $\mathrm{CD} 8^{+} \mathrm{T}$ cell counts in treated animals (Supplemental Figure 2). Results showed that $\alpha$ TIGIT Ab failed to significantly affect 7-day survival in 
A
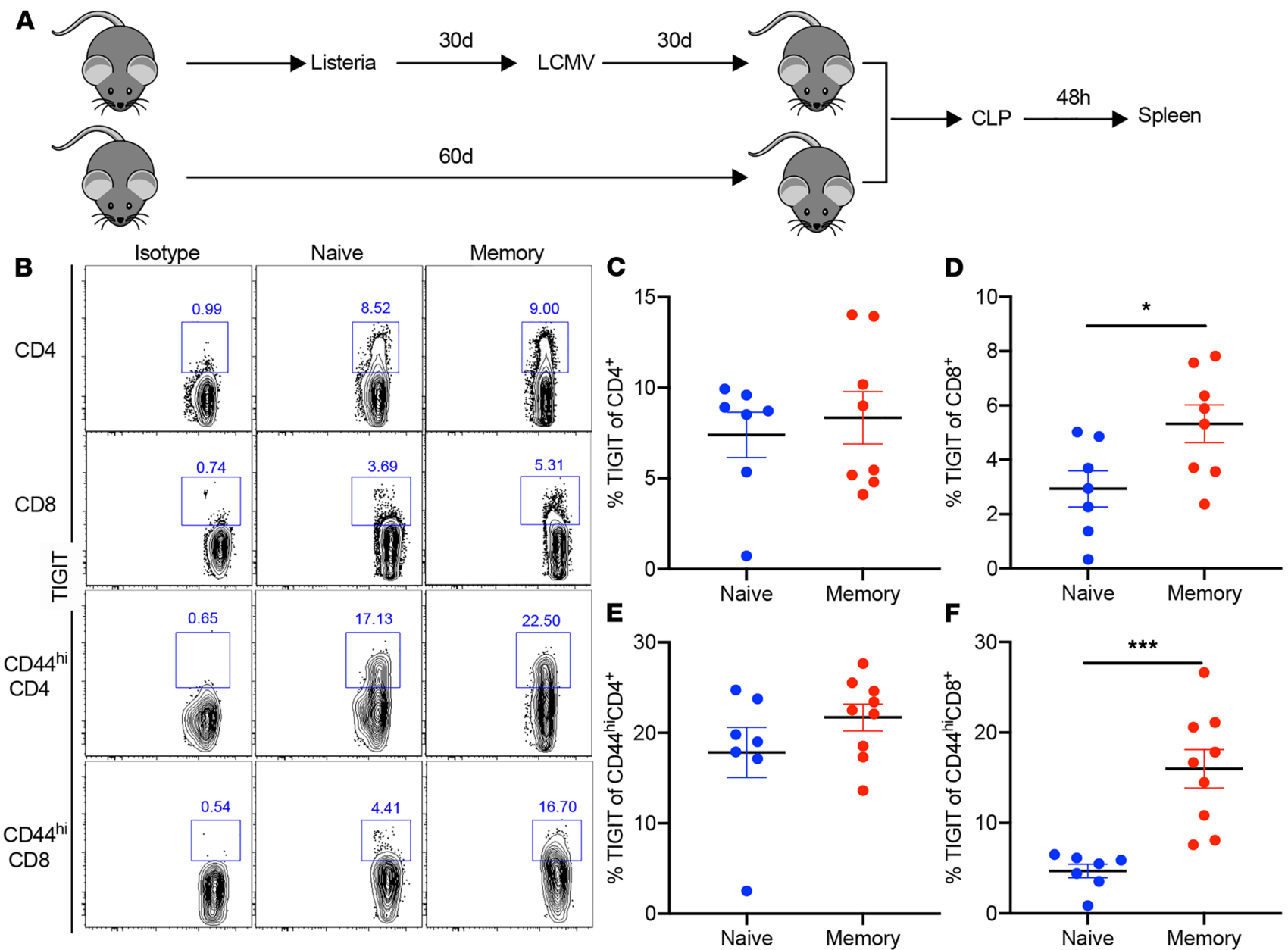

Figure 1. Coinhibitory molecule T cell Ig with ITIM domain expression is higher on CD8 ${ }^{+} \mathrm{T}$ cells in memory versus previously naive mice during sepsis. Naive B6 mice were infected with Listeria monocytogenes (LM) and with lymphocytic choriomeningitis virus (LCMV) i.p. 30 days later. Age-matched naive mice were used as controls. B6 naive and memory mice received cecal ligation and puncture (CLP) and were sacrificed at 48 hours after surgery. (A) Spleens were harvested and coinhibitory molecule T cell Ig with ITIM domain (TIGIT) expression on T cells was determined by flow cytometry. (B) Representative flow plots of TIGIT expression on bulk CD4+, $C D 8^{+}, C D 44^{\text {hi }} \mathrm{CD} 4^{+}$, and $\mathrm{CD} 44^{\text {hic }} \mathrm{CD} 8^{+} \mathrm{T}$ cells in previously naive and memory septic mice. (C and $\left.\mathbf{D}\right)$ Summary data of the percentage of TIGIT on splenic CD4+ and CD8 ${ }^{+}$T cells in previously naive and memory mice ( $n=7-8 /$ group). (E and F) Summary data of the percentage of TIGIT on CD44 hi CD4+ and CD44 hi CD8 ${ }^{+}$T cells in spleens at 48 hours after CLP in previously naive versus memory mice ( $n=7-8 /$ group). Two groups were compared with the Mann-Whitney $U$ test. ${ }^{*} P<0.05,{ }^{* *} P<0.001$. All data are shown as mean \pm SEM and were pooled from 2 independent experiments.

previously naive septic mice (Figure 2B). Given the previous finding that TIGIT expressed on T cells was increased in memory septic mice compared with their previously naive counterparts, we hypothesized that treatment with $\alpha$ TIGIT Ab might confer a survival benefit in memory mice following sepsis. Memory mice underwent CLP and received either $\alpha$ TIGIT Ab or isotype control at 12 and 24 hours after CLP (Figure 2C). Strikingly, septic memory mice treated with aTIGIT exhibited dramatically decreased survival relative to isotype-treated mice $(P=0.0012$, Figure 2D). Almost all memory mice treated with $\alpha$ TIGIT Ab died in the first 3 days following CLP.

Apoptosis of memory $T$ cells is increased by $\alpha$ TIGIT Ab in memory septic mice. To further investigate the sharp early mortality in $\alpha$ TIGIT-treated memory septic mice, we first assessed apoptosis in the splenic $\mathrm{T}$ cell compartment in previously naive versus memory mice treated with $\alpha$ TIGIT Ab or isotype control at 48 hours following CLP (Figure 3A). Annexin V staining permits the detection of phosphatidylserine exposure on the cell membrane of apoptotic cells (19). Simultaneous staining of $\mathrm{T}$ cells with annexin $\mathrm{V}$ and 7-AAD allows the discrimination of early apoptotic (annexin $\mathrm{V}^{+}$7-AAD ${ }^{-}$) and late apoptotic or necrotic cells. Results showed that $\mathrm{CD} 44^{\mathrm{hi}} \mathrm{CD} 4^{+} \mathrm{T}$ cells in memory mice treated with $\alpha \mathrm{TIGIT}$ Ab contained a higher frequency of early apoptotic $\mathrm{T}$ cells relative to isotype-treated memory controls during sepsis $(20.6 \% \pm 1.5 \%$ vs. $13.9 \% \pm 0.7 \%, P=0.0009$, Figure $3 \mathrm{~B})$. In contrast, $\alpha$ TIGIT Ab had no effect 
A
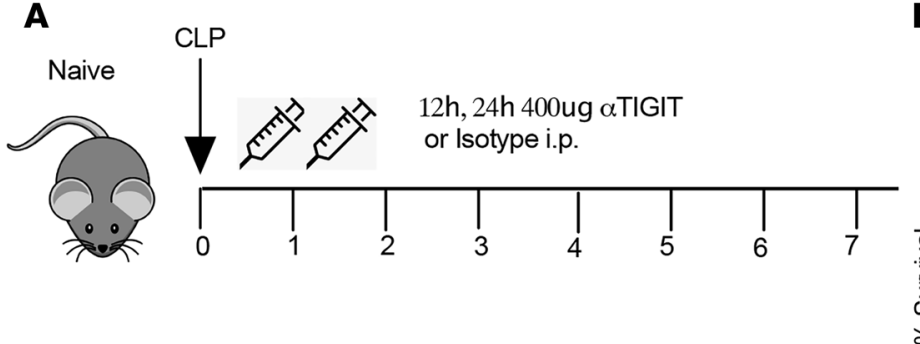

B

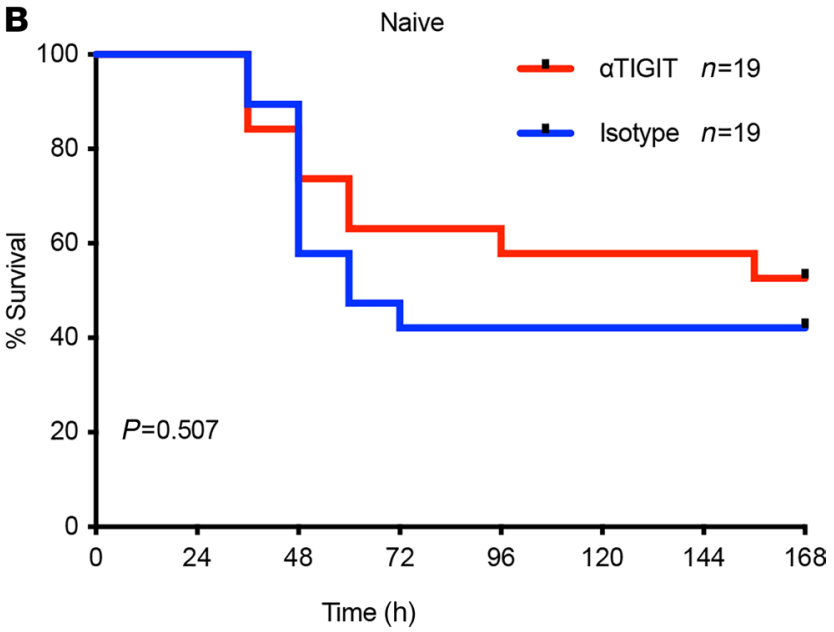

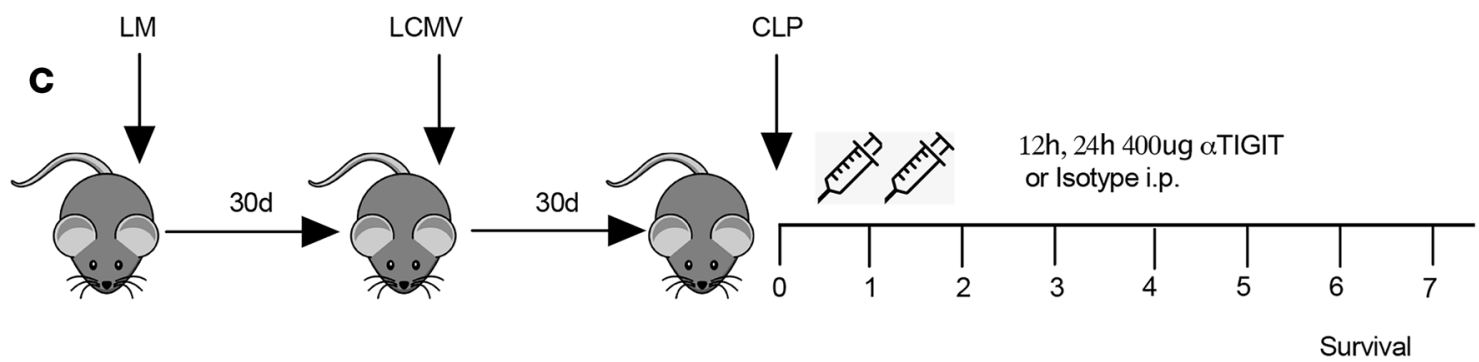

D

Memory

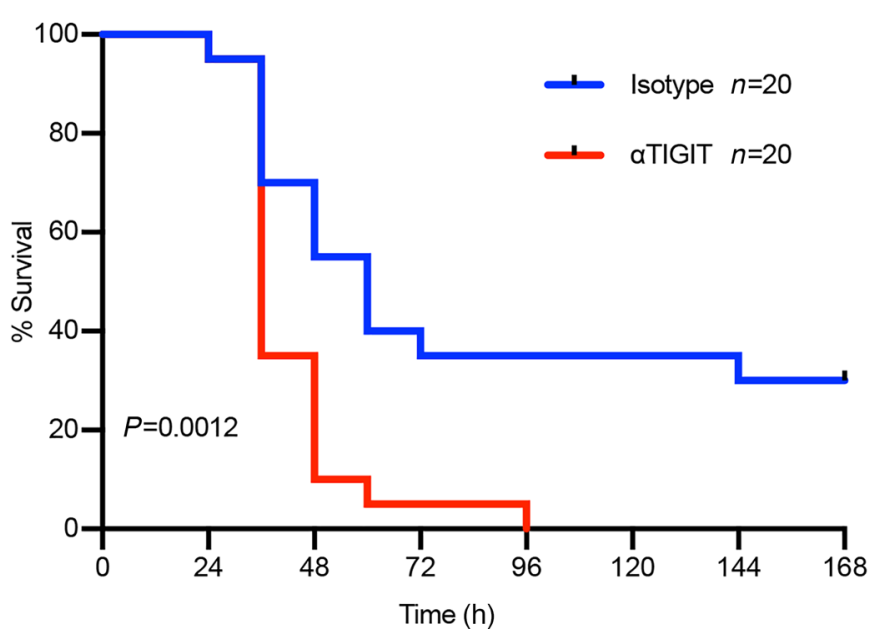

Figure 2. $\alpha$ TIGIT Ab significantly worsens sepsis survival in memory but not previously naive mice. (A) Schematic of experiment setup for CLP sepsis study in naive mice. (B) All naive mice received CLP surgery and were randomized to receive either $\alpha$ TIGIT Ab $(n=19)$ or isotype Ab ( $n=19)$ at specified time points. All animals were monitored for 7-day survival. (C) Schematic of experiment setup for memory septic mice. (D) All memory mice received CLP surgery and were randomized to receive either $\alpha$ TIGIT Ab $(n=20)$ or isotype $A b(n=20)$ at 12 and 24 hours after surgery. All animals were monitored for 7-day survival. Results represent 2 independent experiments. The log-rank (Mantel-Cox) test was used to test for significance.

on the apoptosis of $\mathrm{CD} 44^{10} \mathrm{CD} 4^{+} \mathrm{T}$ cells in either previously naive or memory mice with sepsis (Figure 3C). Within the $\mathrm{CD} 8^{+} \mathrm{T}$ cell compartment, $\alpha$ TIGIT resulted in increased frequencies of apoptotic cells among $\mathrm{CD} 44^{\text {hi }} \mathrm{CD} 8^{+} \mathrm{T}$ cells relative to isotype control in memory but not previously naive septic mice $(18.4 \% \pm 1.9 \%$ vs. $13.4 \% \pm 0.9 \%, P=0.041$, Figure $3, \mathrm{D}$ and $\mathrm{E})$. Conversely, there were no differences in the apoptosis of $\mathrm{CD} 44^{\text {1o }}$ naive $\mathrm{CD} 8^{+} \mathrm{T}$ cells among the 4 groups (Figure $3 \mathrm{~F}$ ). Although memory septic animals possessed increased frequencies of late apoptotic (annexin $\mathrm{V}^{+} 7-\mathrm{AAD}^{+}$) cells among both $\mathrm{CD} 4^{+}$ and $\mathrm{CD} 8^{+} \mathrm{CD} 44^{\mathrm{hi}}$ memory $\mathrm{T}$ cells, frequencies of late apoptotic cells were not affected by anti-TIGIT in either the memory or previously naive septic mice (Supplemental Figure 3). 
$\alpha T I G I T$ Ab decreases frequencies of cytokine-producing $T$ cells in memory septic mice. To further investigate the effect of $\alpha$ TIGIT on T cell function, we assessed the frequencies of TNF- and IFN- $\gamma$ secreting CD4 ${ }^{+}$ and $\mathrm{CD}^{+} \mathrm{T}$ cells in memory versus previously naive mice treated with $\alpha$ TIGIT Ab or isotype control at 48 hours following CLP. Intracellular cytokine staining following ex vivo restimulation revealed that memory septic mice possessed increased frequencies of high-quality multipotent $\mathrm{TNF}^{+} \mathrm{IFN}-\gamma^{+}$-producing $\mathrm{CD} 44^{\text {hi }} \mathrm{CD} 4^{+} \mathrm{T}$ cells compared with previously naive septic mice. Further, whereas $\alpha \mathrm{TIGIT}$ Ab had no effect on the frequencies of $\mathrm{TNF}^{+} \mathrm{IFN}-\gamma^{+}$-producing cells among $\mathrm{CD} 44^{\mathrm{hi}} \mathrm{CD} 4^{+} \mathrm{T}$ cells in previously naive septic mice, it significantly decreased the frequencies of $\mathrm{TNF}^{+} \mathrm{IFN}-\gamma^{+}$-producing cells among CD44 ${ }^{\text {hi }} \mathrm{CD} 4^{+}$ $\mathrm{T}$ cells in memory septic mice (Figure $4, \mathrm{~A}$ and $\mathrm{B}$ ). In contrast, $\alpha$ TIGIT Ab had no effect on the frequencies of $\mathrm{TNF}^{+} \mathrm{IFN}-\gamma^{+}$-producing cells within the $\mathrm{CD} 44^{\text {lo }} \mathrm{CD}^{+} \mathrm{T}$ cell compartment in either previously naive or memory septic mice (Figure $4 \mathrm{C}$ ). With regard to the $\mathrm{CD} 8^{+}$compartment, memory septic mice also possessed higher frequencies of $\mathrm{TNF}^{+} \mathrm{IFN}-\gamma^{+}$-producing $\mathrm{CD} 44^{\mathrm{hi}} \mathrm{CD} 8^{+} \mathrm{T}$ cells relative to previously naive septic mice. Interestingly, although $\alpha$ TIGIT Ab reduced the frequencies of $\mathrm{TNF}^{+} \mathrm{IFN}-\gamma^{+} \mathrm{CD} 44^{\mathrm{hi}} \mathrm{CD} 8^{+} \mathrm{T}$ cells in memory septic mice, it paradoxically increased the frequencies of $\mathrm{TNF}^{+} \mathrm{IFN}-\gamma^{+}$-producing $\mathrm{CD} 44^{\text {hi }} \mathrm{CD} 8^{+} \mathrm{T}$ cells in naive septic mice (Figure 4, D and E). Memory septic mice also possessed increased frequencies of $\mathrm{TNF}^{+} \mathrm{IFN}-\gamma^{+}$-producing cells among $\mathrm{CD} 44^{10} \mathrm{CD} 8^{+} \mathrm{T}$ cells compared with previously naive septic mice, and $\alpha$ TIGIT Ab decreased the frequencies of these CD $44^{\text {lo }} \mathrm{CD}^{+} \mathrm{TNF}^{+} \mathrm{IFN}-\gamma^{+}$cells in memory septic mice (Figure $4 \mathrm{~F})$. Taken together, these data indicate that $\alpha$ TIGIT Ab inhibited cytokine effector function of both $\mathrm{CD}^{+}$and $\mathrm{CD}^{+} \mathrm{CD} 44^{\mathrm{hi}} \mathrm{T}$ cells in memory septic mice, but not in previously naive septic mice.

$\alpha$ TIGIT results in decreased DNAM expression on $C D 44^{\text {hi }} C D 8^{+} T$ cells in memory but not previously naive septic mice. Next, we sought to explore the expression of DNAM, an activating receptor on T cells that competes with TIGIT for the same set of ligands, on T cells isolated from memory versus previously naive septic mice treated with either $\alpha$ TIGIT Ab or isotype control. No change in DNAM expression on either $\mathrm{CD} 44^{\text {hi }} \mathrm{CD} 4{ }^{+}$or $\mathrm{CD} 44^{\text {lo }} \mathrm{CD} 4{ }^{+} \mathrm{T}$ cells was observed among any of the 4 groups (Figure $5, \mathrm{~A}$ and $\mathrm{B}$ ). In contrast, $\alpha$ TIGIT Ab resulted in decreased DNAM expression on $\mathrm{CD} 44^{\mathrm{hi}} \mathrm{CD} 8^{+} \mathrm{T}$ cells, but not $\mathrm{CD} 44^{\mathrm{lo}} \mathrm{CD} 8^{+} \mathrm{T}$ cells in memory septic mice (Figure 5, C and D). In contrast, there was no significant effect of $\alpha$ TIGIT Ab on DNAM expression on either CD $44^{\text {hi }}$ or $\mathrm{CD} 44^{\text {lo }} \mathrm{CD}^{+} \mathrm{T}$ cells in naive septic mice (Figure $5, \mathrm{C}$ and $\mathrm{D}$ ).

Given the synergistic nature of coinhibitory receptors, PD-1, 2B4, and TIM3 expression on memory and naive $\mathrm{T}$ cells were also assessed in memory and previously naive septic mice treated with $\alpha$ TIGIT or isotype control. PD-1 expression was increased on both $\mathrm{CD} 44^{\mathrm{hi}} \mathrm{CD} 4^{+}$and $\mathrm{CD} 44^{\mathrm{hi}} \mathrm{CD} 8^{+} \mathrm{T}$ cells in memory versus previously naive septic mice treated with $\alpha$ TIGIT Ab (Supplemental Figure 4, A, B, D, and E). No changes in $\mathrm{PD}-1$ expression on $\mathrm{CD} 44^{1 \circ} \mathrm{CD} 4^{+}$or $\mathrm{CD} 44^{10} \mathrm{CD} 8^{+} \mathrm{T}$ cells were observed among the 4 groups (Supplemental Figure 4, C and F). Additionally, there were no significant differences in 2B4 or TIM-3 expression on memory or naive T cells among any of 4 groups (data not shown).

Foxp $3^{+}$Tregs from $\alpha$ TIGIT-treated memory mice exhibit reduced activation and differentiation. We next sought to characterize the effect of $\alpha$ TIGIT Ab on the differentiation and activation of Tregs in both memory and previously naive mice at 48 hours after CLP. Although there was no significant effect of $\alpha$ TIGIT Ab on the percentage and absolute numbers of Foxp $3^{+}$Tregs in either memory or previously naive groups (Figure 6, A and B), several aspects of Treg activation and differentiation were altered in anti-TIGIT-treated memory septic mice. First, the Ikaros family transcription factor Helios, which has been shown to be indicative of more stable and functional Tregs (20), was not affected in Tregs isolated from previously naive septic mice. In contrast, $\alpha$ TIGIT resulted in a significant decrease in the frequency of Helios of Foxp3 $3^{+}$Tregs in memory septic mice compared with isotype-treated memory controls (Figure 6, C and D). Cytotoxic T lymphocyte antigen 4 (CTLA-4) has been reported to be critically required for the function of Tregs in vivo (21). Our data demonstrated that Tregs isolated from $\alpha$ TIGIT-treated memory septic mice exhibit decreased CTLA-4 expression relative to Tregs isolated from $\alpha$ TIGIT-treated previously naive septic mice (Figure $6 \mathrm{E}$ ).

The effect of $\alpha$ TIGIT on the activation of Tregs was also evaluated in both memory and naive mice. Memory septic mice possessed fewer frequencies of $\mathrm{CD} 69^{+} \mathrm{Foxp} 3^{+} \mathrm{CD} 4^{+}$Tregs relative to previously naive animals with sepsis, and treatment with $\alpha$ TIGIT had no effect on the CD69 expression of Tregs in memory septic mice at 48 hours after CLP (Figure 6F). Further, CD62L has been shown to be indicative of the resting status of Tregs (22). Strikingly, whereas $\alpha$ TIGIT significantly decreased CD62L expression on Tregs in previously naive mice with sepsis, it significantly increased CD62L expression on Tregs in memory mice with sepsis (Figure 6G). To address another major functional readout of Treg suppression, we assessed 
A
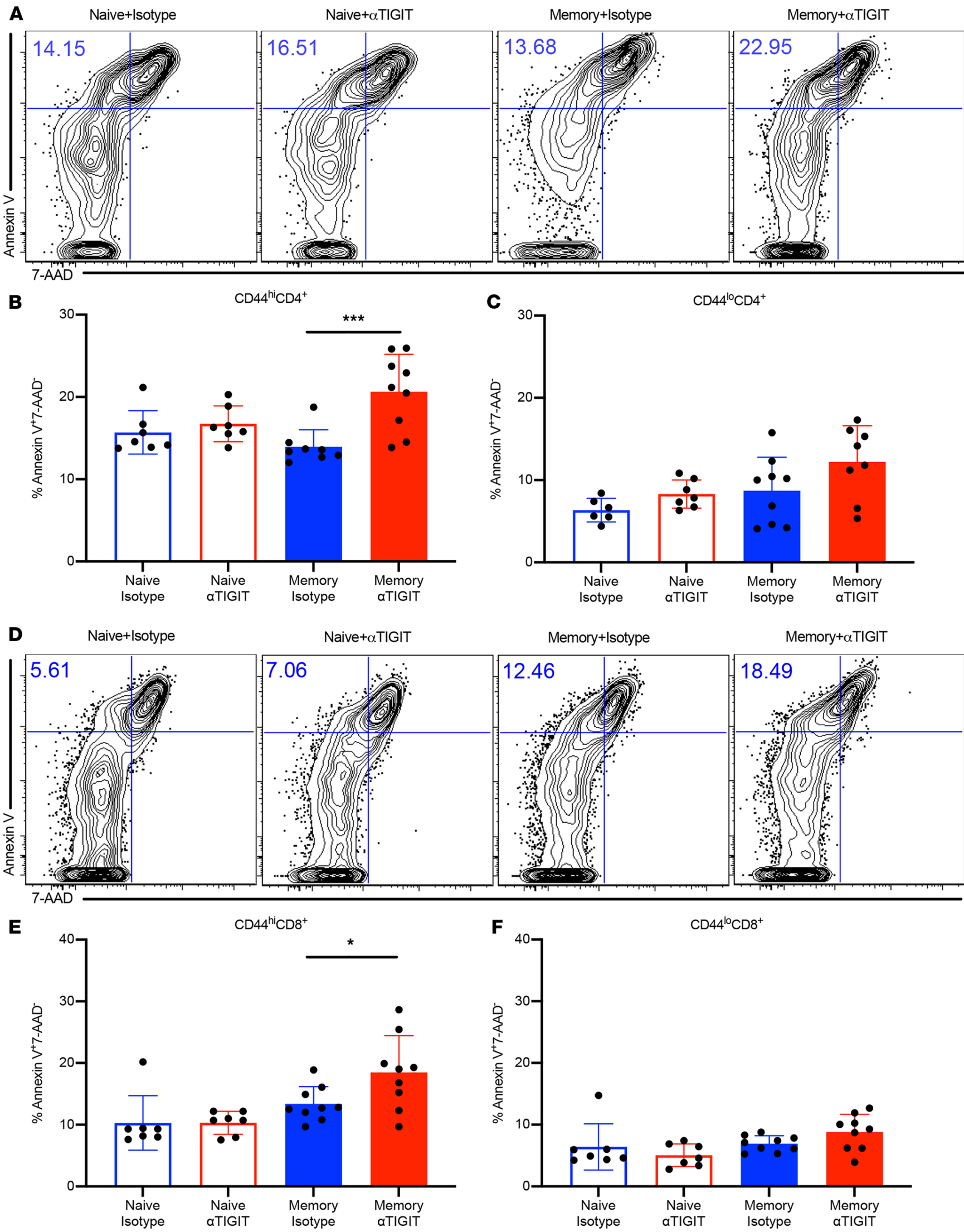
Figure 3. Apoptosis of CD44 ${ }^{\text {hi }}$ memory T cells is accelerated by $\alpha$ TIGIT Ab in memory but not previously naive septic mice. Memory mice and agematched naive controls received CLP, followed by injection of $\alpha$ TIGIT Ab or isotype control Ab at 12 and 24 hours after CLP. Mice were sacrificed and spleens were harvested at 48 hours after CLP. Splenocytes were stained with annexin $V$ and 7-AAD for T cell apoptosis by flow cytometry. (A) Representative flow plots for annexin $\mathrm{V}^{+}$and 7-AAD- staining gated on $\mathrm{CD} 44^{\text {hi }} \mathrm{CD} 4^{+} \mathrm{T}$ cells. (B and $\mathbf{C}$ ) Summary data depicting frequency of apoptotic (annexin $\mathrm{V}^{+} 7-\mathrm{AAD}^{-}$) $\mathrm{CD} 44^{\text {hi }} \mathrm{CD} 4^{+}$and $\mathrm{CD} 44^{10} \mathrm{CD} 4^{+} \mathrm{T}$ cells in previously naive versus memory mice treated with $\alpha$ TIGIT Ab or isotype Ab ( $n=7-9 /$ group). (D) Representative flow plots for annexin $\mathrm{V}^{+}$and 7-AAD- staining gated on $\mathrm{CD} 44^{\text {hi }} \mathrm{CD} 8^{+} \mathrm{T}$ cells. (E and $\mathbf{F}$ ) Summary data of frequency of apoptotic CD44 ${ }^{\text {hi }} \mathrm{CD} 8^{+}$and $C D 44^{\mathrm{o}} \mathrm{CD} 8^{+} \mathrm{T}$ cells among the 4 groups ( $n=7-9 /$ group). Groups were compared using 1-way ANOVA analysis and Tukey's multiple comparison test. ${ }^{*} P<0.05,{ }^{* * *} P<0.001$.

IL-10 secretion by Tregs that were isolated from memory septic mice treated with either isotype control or anti-TIGIT. Results indicated that Foxp $3^{+}$Tregs isolated from anti-TIGIT-treated memory septic mice contained significantly fewer IL-10 producers relative to Foxp $3^{+}$Tregs isolated from isotype-treated memory septic mice (Figure $6 \mathrm{H}$ ). Taken together, these data indicate that $\alpha$ TIGIT results in less stable and less functional Tregs and inhibited the activation of Tregs in memory septic mice, while having no effect or the opposite effect on Tregs in previously naive septic mice.

$\alpha$ TIGIT Ab differentially affected bacterial load and cytokines in peritoneal fluid in memory versus previously naive septic mice. To determine the effect of $\alpha$ TIGIT Ab on bacterial clearance in the infectious site in memory versus previously naive mice with sepsis, we examined the bacterial load in peritoneal fluid (PF) at 36 hours after CLP. The 36-hour time point was chosen to capture relevant changes in systemic cytokines. Relative to previously naive septic mice, memory septic mice possessed significantly less bacterial load in the PF during sepsis. Further, whereas treatment with $\alpha$ TIGIT had no effect on bacterial load in memory septic mice, it significantly decreased bacterial load in the PF of previously naive mice at 36 hours after CLP (Figure 7A). Levels of circulating systemic inflammatory (IL1 $\beta$, IL-6, TNF, IL-2, IL-13, and MCP-1) as well as antiinflammatory (IL-10) cytokines were also evaluated 36 hours after CLP in memory versus previously naive mice. Compared with isotype-treated previously naive controls, IL-6, IL-10, and MCP-1 serum levels exhibited a trend toward increase in $\alpha$ TIGIT-treated previously naive mice (IL-6: $P=0.13$; IL-10: $P=0.13$; and MCP-1: $P=0.07$ ). Although serum IL-10, IL-6, and MCP-1 were lower in the anti-TIGIT-treated memory mice relative to anti-TIGIT-treated previously naive mice (Figure 7, B-D), results indicated no significant differences in the anti-TIGIT-treated versus isotype control-treated memory septic animals in any of the cytokines tested (Figure 7, B-D, and data not shown). Moreover, analysis of an additional time point at 48 hours after CLP revealed no significant differences between the anti-TIGIT-treated versus control groups in either the previously naive or memory septic animals in any of the cytokines tested (Supplemental Figure 5). Further, there were no statistically significant differences in the absolute numbers of $\mathrm{CD} 4^{+}$or $\mathrm{CD} 8^{+} \mathrm{T}$ cell populations (Supplemental Figure 6A), or in any of the innate immune subsets $\left(C D 11 \mathrm{c}^{+} \mathrm{DCs}, \mathrm{CD} 11 \mathrm{~b}^{+}\right.$myeloid cells, $\mathrm{F} 4 / 80^{+}$macrophages, NK1.1 $1^{+} \mathrm{NK}$ cells, or Gr-1 ${ }^{+}$neutrophils, Supplemental Figure $6 \mathrm{~B}$ ) within the PF of anti-TIGIT- versus isotype-treated memory mice. There were also no differences in the absolute

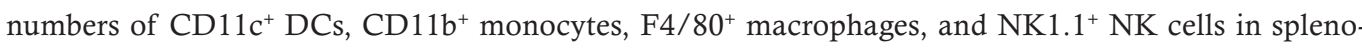
cytes of anti-TIGIT-treated versus control septic memory mice (Supplemental Figure 6C).

\section{Discussion}

In the present study, we demonstrate fundamental differences in the role of TIGIT coinhibitory signaling during sepsis in previously naive versus immunologically experienced murine hosts. Consistent with a previous report, our data demonstrated that TIGIT was upregulated on memory $\mathrm{T}$ cells both before and during sepsis (6). Naive mice possessed fewer memory T cells and exhibited less TIGIT expression on CD4 ${ }^{+}$and $\mathrm{CD}^{+} \mathrm{T}$ cells (Supplemental Figure 1, H and I). We submit that this immunologically experienced mouse model, generated via sequential infection of mice with acutely cleared bacterial and viral pathogens, better recapitulates relevant aspects of the human immune system. Thus, the fact that $\alpha$ TIGIT Ab had no impact on the survival in previously naive septic mice, whereas it sharply worsened survival in memory mice with sepsis, has important implications for the role of TIGIT during human immune responses in sepsis. Mechanistically, $\alpha$ TIGIT increased apoptosis and decreased cytokine effector function of CD $44^{\text {hi }} \mathrm{T}$ cells in memory but not previously naive mice. Additionally, $\alpha$ TIGIT diminished the stability and activity of Tregs during sepsis in memory mice, but not in previously naive septic mice.

TIGIT has emerged as a crucial coinhibitory receptor in studies of antitumor and antiviral immune responses $(23,24)$. An initial study demonstrated that TIGIT exerted immunosuppressive effects by 

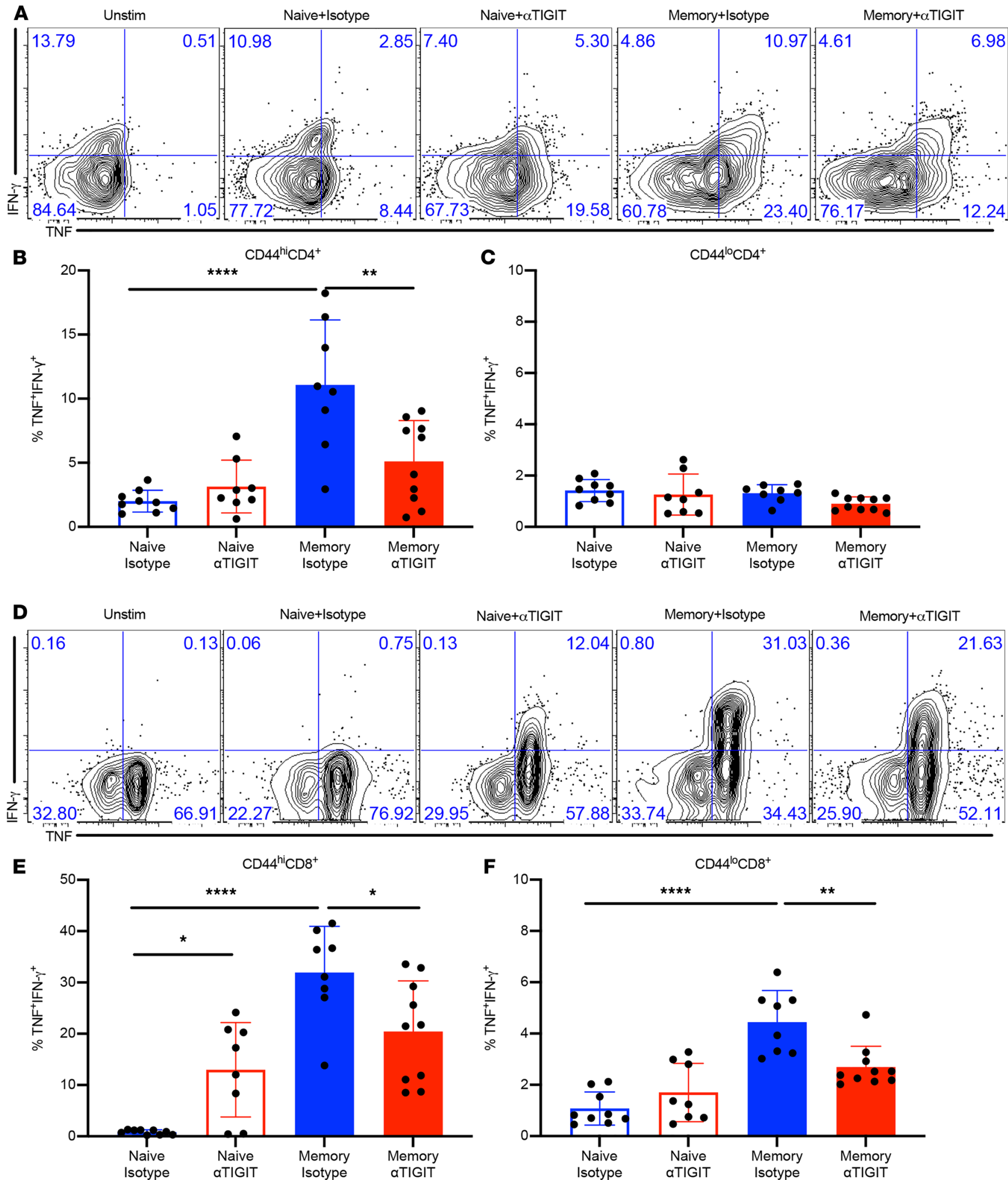

Figure 4. $\alpha$ TIGIT Ab decreases frequencies of cytokine-producing T cells in memory septic mice. Both naive and memory mice were subjected to CLP, received $\alpha$ TIGIT Ab or isotype control at 12 and 24 hours after CLP, and were sacrificed at 48 hours after CLP. Splenocytes were harvested and stimulated ex vivo with PMA and ionomycin for 4 hours, and then were assessed for TNF, IFN- $\gamma$ production via intracellular cytokine staining. (A) Representative flow plots for TNF ${ }^{+}$IFN- $\gamma^{+}$staining of CD44 hiCD4 ${ }^{+} T$ cells. (B and C) Summary figures for CD4 ${ }^{+} T$ cell cytokine staining ( $n=9-10 /$ group). (D) Representative flow plots for TNF ${ }^{+}$IFN- $\gamma^{+}$staining of CD44 hi CD8 ${ }^{+} T$ cells. (E and F) Summary figures for CD44 $4^{\text {hi }} C D 4^{+}$and $C D 44^{\text {hic }} C D 8^{+}$T cell cytokine staining in 4 groups ( $n$ = 8-10/group). Results represent a minimum of 2 independent experiments. Groups were compared using 1-way ANOVA analysis and Tukey's multiple comparison test. ${ }^{*} P<0.05,{ }^{* *} P<0.01,{ }^{* * *} P<0.0001$. 
A

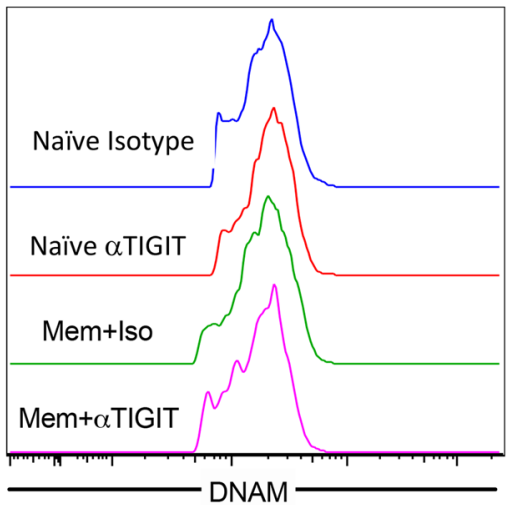

CD44hicD4

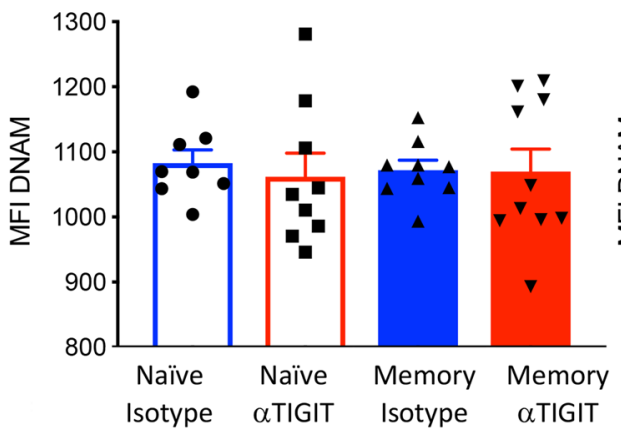

C

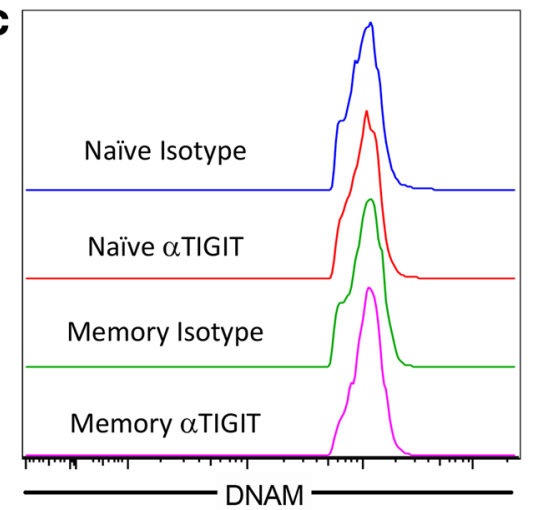

CD44 ${ }^{\text {hi }}$ CD8

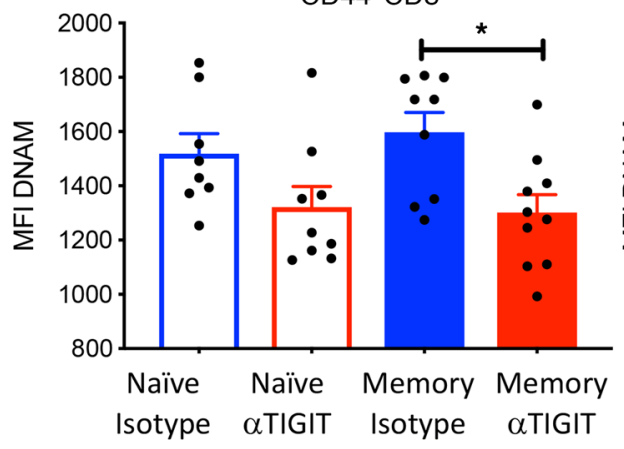

B
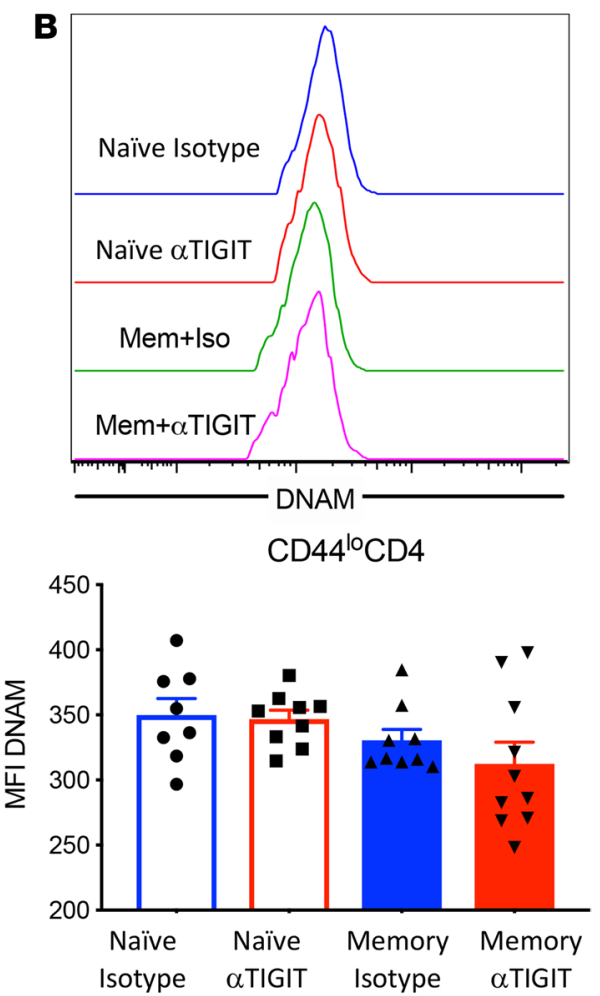

D

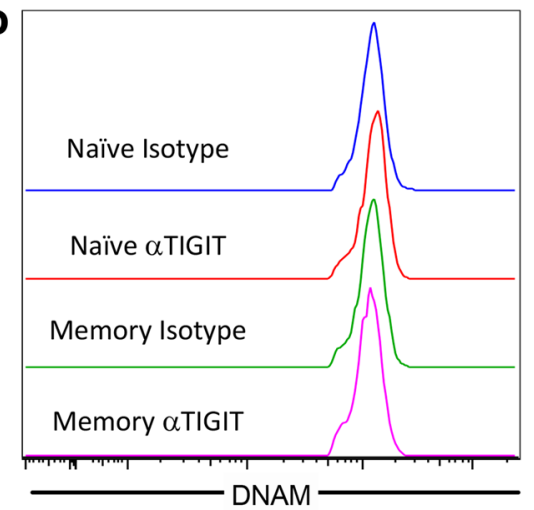

CD44 ${ }^{\circ} \mathrm{CD} 8$

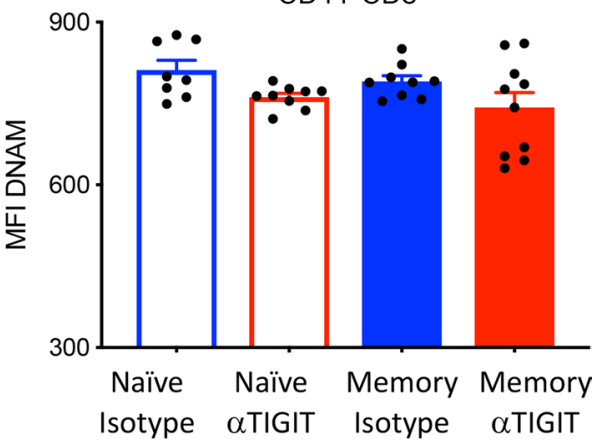

Figure 5. Memory $\mathrm{CD8}^{+} \mathrm{T}$ cells exhibit decreased DNAM expression in $\alpha \mathrm{TICIT}$-treated memory mice relative to memory controls. Memory and naive mice were subjected to CLP and received 2 doses of $\alpha$ TIGIT Ab or isotype control at 12 and 24 hours after CLP. Spleens were harvested at 48 hours after CLP and DNAM expression on T cells was assessed by flow cytometry. (A and $\mathbf{B}$ ) Representative flow histograms and summary data depicting DNAM expression on CD44 ${ }^{\text {hi }} \mathrm{CD} 4^{+}$and $\mathrm{CD} 44^{\mathrm{l}} \mathrm{CD} 4^{+} \mathrm{T}$ cells. (C and D) Representative flow histograms and summary figure depicting DNAM MFI of $\mathrm{CD} 44^{\text {hi }} \mathrm{CD}^{+}$and $\mathrm{CD} 44^{10} \mathrm{CD} 8^{+} \mathrm{T}$ cells, $n=8-10$ in each group. Groups were compared using 1-way ANOVA analysis and Tukey's multiple comparison test. ${ }^{*} P<0.05$. 
A

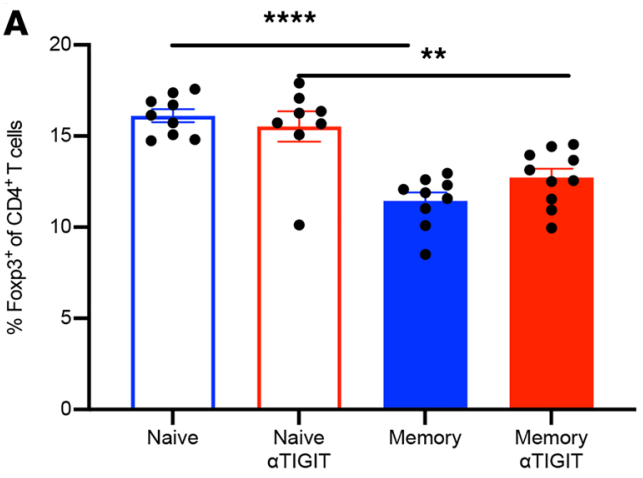

C

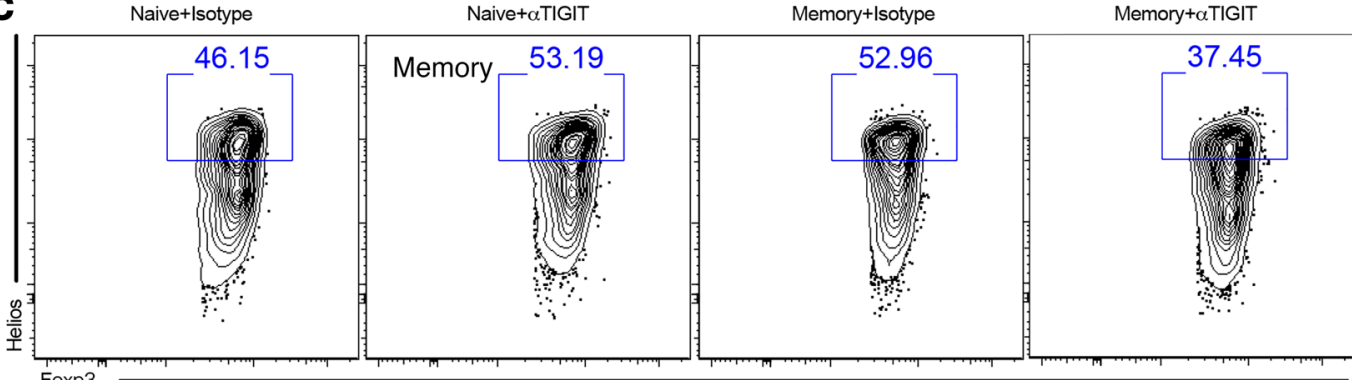

D

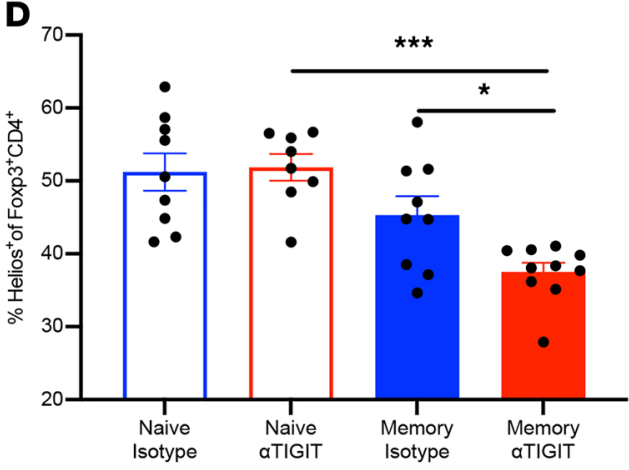

$\mathbf{F}$

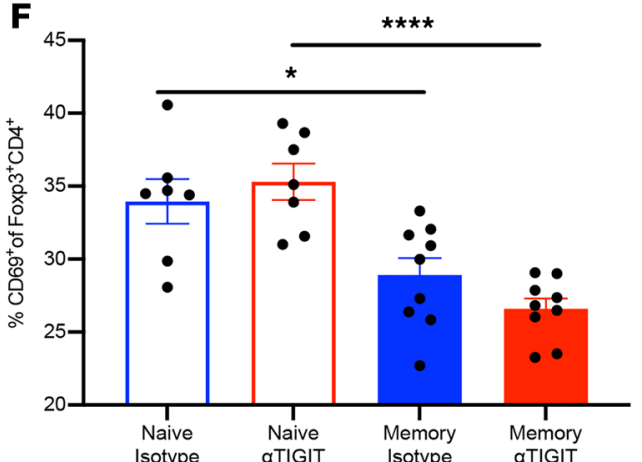

H

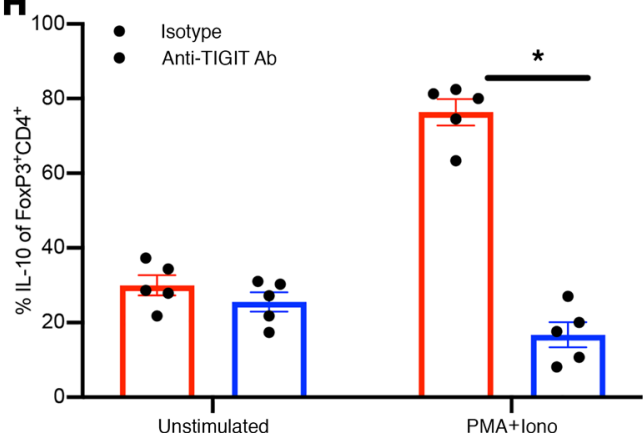

B

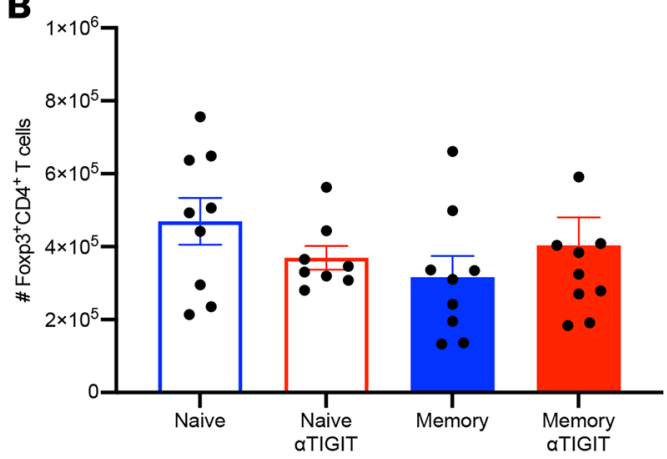

E

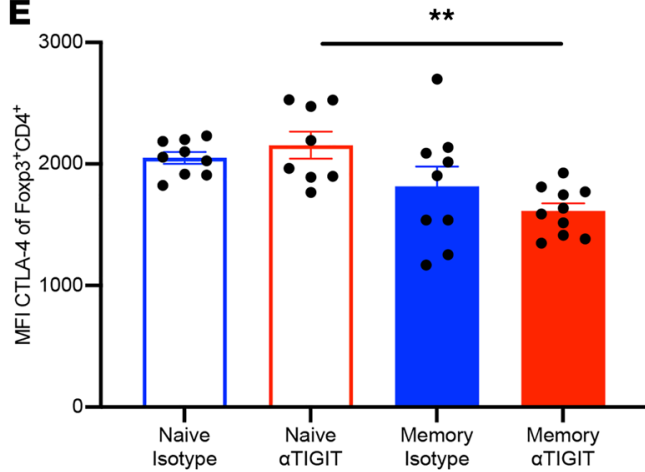

G

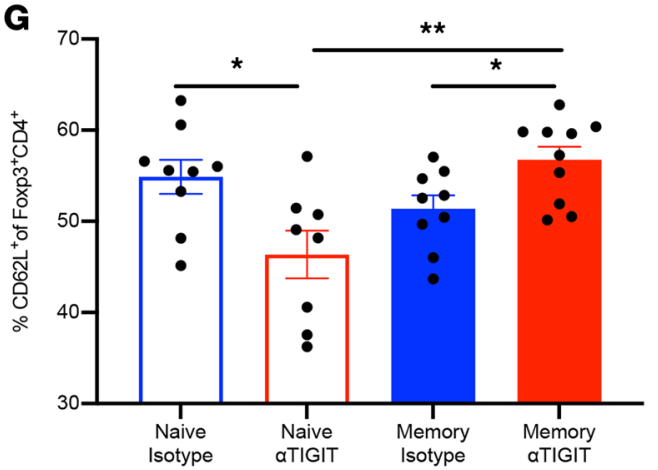

Figure 6. Foxp ${ }^{+}$Tregs from $\alpha$ TICIT-treated memory mice exhibit deteriorated differentiation and activation.

Splenocytes were harvested at 48 hours after CLP from memory and previously naive septic mice treated with aTIGIT or isotype Ab. (A) Summary data of the percentage of Foxp $3^{+}$Tregs in the 4 groups.

(B) Absolute numbers of Foxp $3^{+}$Tregs among 4 groups. $n=8-9 /$ group. Results were representative of 2 independent experiments. Groups were compared using 1-way ANOVA analysis and Tukey's multiple comparison test. (c) Representative flow plots depicting Helios expression on $\mathrm{CD}^{+} \mathrm{Foxp}^{+} \mathrm{T}$ cells. (D)

Summary data of the percentage of Helios-expressing cells among Foxp $3^{+}$Tregs. (E) Summary figure depicting CTLA-4 MFI of Foxp $3^{+}$Tregs ( $F$ and $\mathbf{G}$ ) Summary data of the percentage of $\mathrm{CD}^{\circ} 9^{+}$and CD62L $^{+}$cells among Foxp $3^{+}$ Tregs. (H) Splenocytes from memory septic mice were harvested at 48 hours and restimulated with PMA/ionomycin ex vivo for 4 hours and analyzed for IL-10. Results represent 2 independent experiments. Data are shown as the mean \pm SEM. Groups were compared using 1-way ANOVA analysis and Tukey's multiple comparison test. ${ }^{*} P<0.05$, ${ }^{* *} P<0.01$, ${ }^{* *} P<$ $0.001,{ }^{* * *} P<0.0001$. 
triggering CD155 in DCs, thereby preventing DC maturation and inducing IL-10 production (12). Other studies revealed that TIGIT can directly inhibit $\mathrm{T}$ cell proliferation and cytokine production independent of antigen-presenting cells (APCs) (25). Immune suppression characterized by increased expression of coinhibitory receptors plays a crucial role in the immunopathological changes of sepsis, which results in secondary infection and worse outcome (26). Recently, checkpoint blockade therapies have garnered attention in the area of clinical research. Anti-TIGIT alone or in combination with PD-1 blockade has emerged as a promising therapy in antitumor treatment $(13,27)$. We also demonstrated that anti-TIGIT Ab (clone 1G9) improved survival in septic mice with preexisting malignancy (unpublished observations), a situation characterized by immune activation and development of effector/ memory T cells (28). In the study, results showed that the blockade of TIGIT pathway in naive septic mice improved $\mathrm{T}$ cell function by increasing the frequencies of $\mathrm{TNF}^{+} \mathrm{IFN}-\gamma^{+}$-producing $\mathrm{T}$ cells. This increased $\mathrm{T}$ cell function was associated with improved control of bacterial burden in the PF. Notably, this improved bacterial control was not associated with a survival benefit in previously naive septic mice, suggesting that mortality in this model is mediated by immune dysregulation and not from bacterial overgrowth per se, a concept that has been repeatedly supported in studies of sepsis in mice and humans. What then underlies the observed disparate effects of $\alpha$ TIGIT Ab on sepsis survival in memory versus naive mice? Joller and colleagues demonstrated that TIGIT engagement can limit T cell-driven inflammation and protect against immune pathology via induction of immune-modulatory cytokine IL-10 in acute viral infection model (29). Because memory mice possess more activated memory $\mathrm{T}$ cells with higher frequencies of proinflammatory cytokine producers and are better able to control bacterial load relative to naive mice during sepsis, we posit that the elevated TIGIT expression on memory $\mathrm{T}$ cells would normally function to limit the overactivation of $\mathrm{T}$ cells and immunopathological injury in memory septic mice. Blockade of TIGIT signaling may therefore lead to overactivation of memory $\mathrm{T}$ cells, resulting in activated-induced apoptosis and dysfunction of $\mathrm{T}$ cells, and contributing to worsened survival in memory septic mice. It is important to note that although a recent study in a cancer model attributed the function of anti-TIGIT to its action on NK cells (30), we found no differences in the number of NK cells between the isotype versus anti-TIGIT-treated groups for either previously naive or memory septic animals (data not shown).

Although TIGIT is expressed at higher frequency among $\mathrm{CD}^{+} \mathrm{T}$ cells, the difference in frequency of TIG$\mathrm{IT}^{+}$cells between naive and memory mice is more pronounced among $\mathrm{CD} 8^{+} \mathrm{T}$ cell populations. These data suggest that $\mathrm{TIGIT}^{+} \mathrm{CD} 8^{+} \mathrm{T}$ cells may represent a specific differentiation program, and that TIGIT could be playing a cell autonomous role on these cells. For example, our data also demonstrated that $\alpha$ TIGIT resulted in decreased DNAM expression on $\mathrm{CD}^{+} \mathrm{T}$ cells, which itself could lead to increase mortality in memory mice with sepsis. This possibility is consistent with a recent study that showed $\mathrm{CD} 226^{\text {hi }} \mathrm{CD} 8^{+} \mathrm{T}$ cells are required for the efficacy of anti-TIGIT immunotherapy in a tumor model (31). In our study, although $\alpha$ TIGIT affected the expression of this costimulatory receptor, it had no effect on the expression of numerous coinhibitory molecules, including PD-1, 2B4, and TIM-3 on CD44 ${ }^{\text {hi }}$ or CD44 $4^{\text {lo }} \mathrm{T}$ cells either in previously naive or memory mice with sepsis. These findings are in line with studies showing that anti-TIGIT did not significantly impact PD-1 expression by antigen-specific $\mathrm{CD}^{+} \mathrm{T}$ cells in patients with melanoma (32). However, they are in opposition to another report showing that Ab-mediated TIGIT blockade resulted in significant downregulation of PD-1 and TIM-3 expression during chronic viral infection (29). Thus, further investigation into the conditions under which TIGIT blockade affects other cosignaling molecule expression and signaling are required.

In addition to its expression and cell-autonomous effect on effector T cells, TIGIT is also expressed on Tregs, where it serves to promote Treg suppressor function during sepsis (27). Recent work shows that TIGIT expression marks a subset of Tregs that exhibit higher Treg effector molecules (i.e., CTLA-4) and enhanced suppressive capacity in vitro $(33,34)$. Further, Helios expression distinguishes thymus-derived Tregs from peripherally induced ones and is also indicative of more stable and functional Tregs (35). The fact that $\alpha$ TIGIT-induced reduction in Helios expression in Tregs in memory septic mice is associated with increased mortality in sepsis is line with published reports showing that Tregs are beneficial during the early, high-inflammatory stage of sepsis $(36,37)$. Thus, in addition to cell-autonomous effects on memory T cells, anti-TIGIT could be indirectly affecting $\mathrm{T}$ cell apoptosis and effector function through its effect on Treg function, possibly warranting further investigation.

Our study is limited by the fact that we used a model of CLP, a mouse model that is widely thought to approximate the situation of ruptured appendicitis in humans. Because sepsis as it is 
A

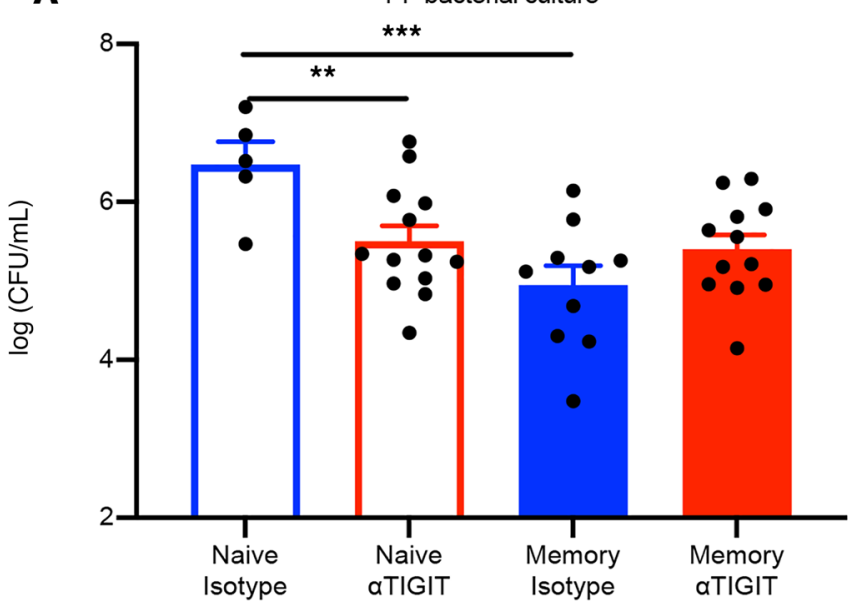

B

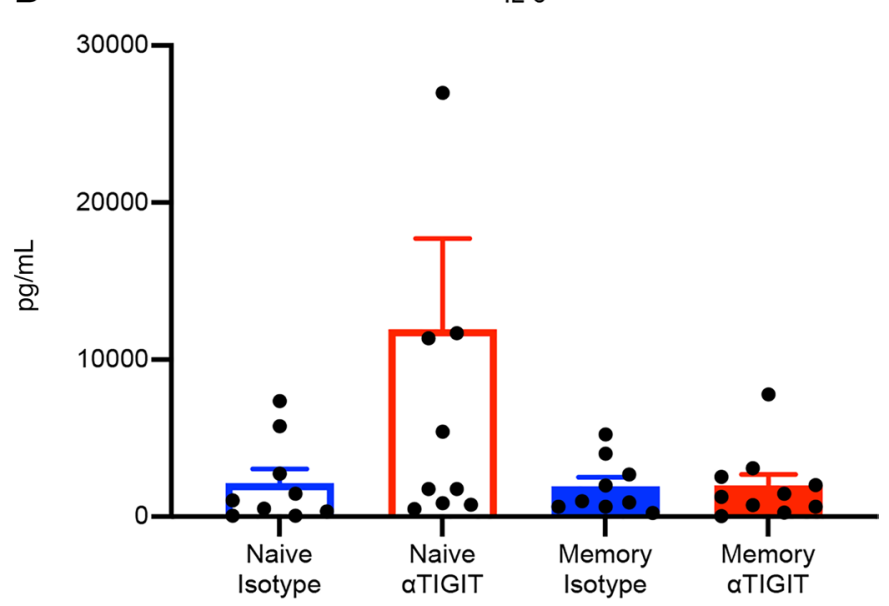

C

IL-10

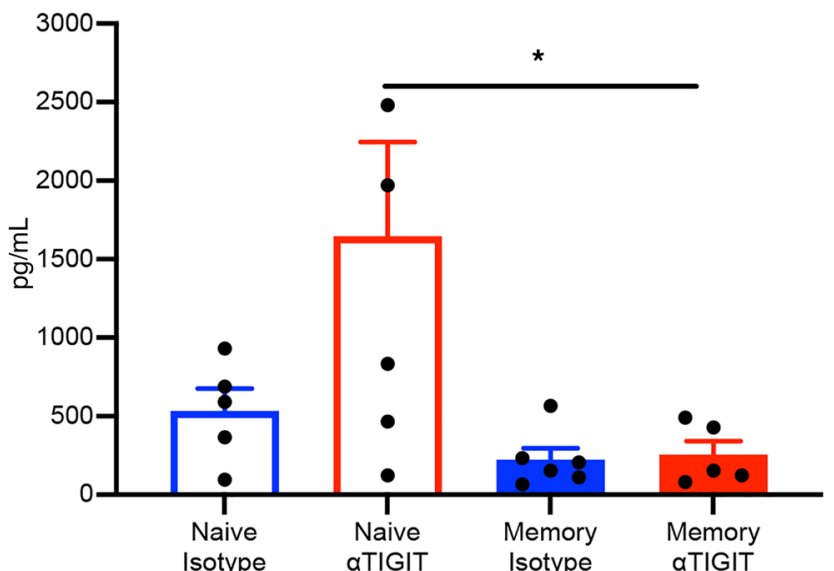

D

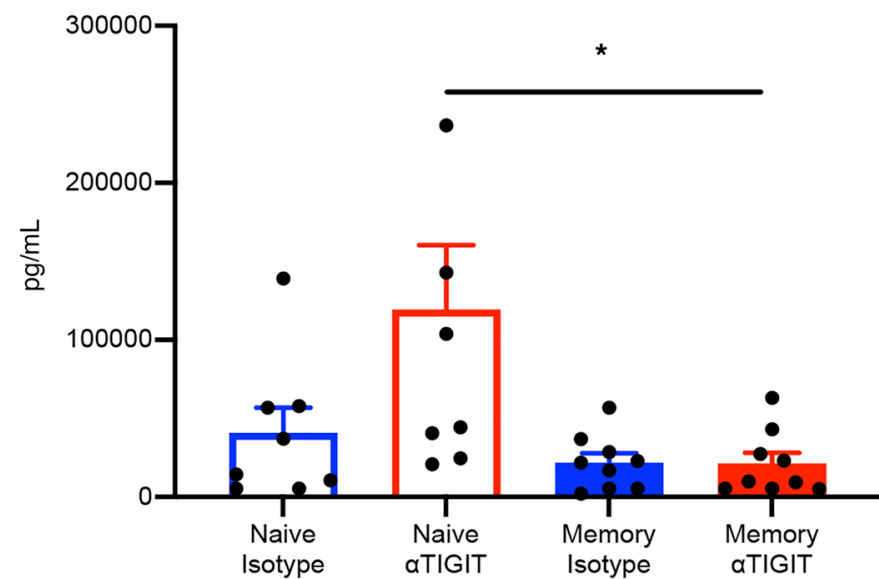

Figure 7. $\alpha$ TIGIT Ab differentially affected bacterial load and cytokines in peritoneal fluid in memory versus previously naive septic mice. Both previously naive and memory septic mice were administered $\alpha$ TIGIT Ab or isotype Ab at12 and 24 hours after CLP, and then were sacrificed at 36 hours after CLP and the sterile peritoneal fluid (PF) was taken for bacterial culture and cytokine detection. (A) Bacterial load in PF was measured in both memory and naive septic mice with or without $\alpha$ TIGIT. (B-D) Summary data of cytokines IL-6, IL-10, and MCP-1 as measured in the PF in the 4 groups. All data are representative of a minimum of 2 independent experiments. Groups were compared using 1-way ANOVA analysis and Tukey's multiple comparison test. ${ }^{*} P<0.05,{ }^{* *} P<0.01,{ }^{* *} P<0.001$.

defined clinically can arise from a number of different infectious etiologies, including respiratory and urinary tract infections, the findings in this model of peritonitis may not be representative of other sepsis etiologies. Indeed, additional work in other models of sepsis, and in human septic patients, will be required to fully elucidate the role of TIGIT in sepsis-induced immune dysregulation.

The differential roles that TIGIT plays in previously naive versus immunologically experienced hosts is an essential consideration for the development of immunotherapeutic approaches involving the TIGIT blockade. Because our data showed that blockade of TIGIT pathway triggered the apoptosis and dysfunction of effector $\mathrm{T}$ cells, as well as dampened the function of Tregs in immunologically experienced host during sepsis, we conclude that rather than promoting $\mathrm{T}$ cell exhaustion and dysfunction like other coinhibitory receptors, TIGIT might play a protective role in preventing $\mathrm{T}$ cell apoptosis and preserving $\mathrm{T}$ cell function during sepsis. Finally, this study sheds light on the complexity and contradiction of targeting coinhibitory receptor during sepsis. Therefore, further investigation is required to determine whether the enhancement of the TIGIT pathway could confer a survival benefit in immunologically experienced hosts during sepsis, and how TIGIT coinhibitory signaling interacts with PD-1, 2B4, and TIM-3 on both effector and Tregs during sepsis. 


\section{Methods}

Memory mice model. Six-week-old male and female C57BL/6J (B6) mice were purchased from The Jackson Laboratory. All animals were housed in the BSL-2 facility of Emory University and maintained following Emory IACUC guidelines (protocol 2003238-082518). For the memory mouse model, $1 \times 10^{4} \mathrm{CFU}$ of L. monocytogenes were i.p. injected. After 30 days, these mice were i.p. injected with a single dose of $2 \times 10^{5} \mathrm{PFU}$ of the Armstrong strain of LCMV. Titers of the virus were determined by plaque assay on Vero cells. The model was established after another 30 days after LCMV. The memory mice generated via this protocol were bled and assessed0, $10,25,40$, and 56 days after L. monocytogenes infection by flow cytometry. Both of these acute infections were cleared on day 59 after L. monocytogenes infection. Age- and sex-matched B6 laboratory mice, termed naive mice, served as controls and were maintained in the same BSL-2 facility for 60 days before surgery.

CLP. CLP was performed 60 days after L. monocytogenes infection. Under isoflurane anesthesia, a midline incision was performed, and the cecum was externalized. The cecum was ligated with a 4-0 silk suture and punctured through and through with a 25 -gauge needle. All animals received buprenorphine $(0.1 \mathrm{mg} / \mathrm{kg}$; McKesson Medical) preoperatively for pain relief. Immediately after surgery, animals received $1 \mathrm{~mL}$ sterile saline for fluid resuscitation as well as antibiotics $(50 \mathrm{mg} / \mathrm{kg}$ ceftriaxone and $35 \mathrm{mg} / \mathrm{kg}$ metronidazole, Sigma-Aldrich) every 12 hours for 2 days. Mice were randomized to receive $400 \mu \mathrm{g}$ anti-TIGIT blocking mAb (clone 1G9, BioXcell) (18) or isotype control Ab (mouse IgG, clone MOPC-21, BioXcell) via i.p. injection at 12 and 24 hours after CLP. All animals were euthanized with $\mathrm{CO}_{2}$ asphyxiation at the indicated time points.

Flow cytometry. Spleens were harvested after mice were sacrificed 48 hours after CLP, and then were processed to single-cell suspensions though a $70 \mu \mathrm{m}$ filter. Splenocytes were rinsed with $10 \mathrm{~mL}$ cold PBS, and $200 \mu \mathrm{L}$ from each spleen was put into a 96-well plate for staining. Anti-CD3 (BD, clone 500A2), anti-CD4 (clone RM4-5, BioLegend), anti-CD8 (clone MCD0830, Invitrogen), anti-CD44 ( clone IM7, BioLegend), anti-CD226 (clone 10E5, BioLegend), anti-PD-1 (clone 29F.1A12, BioLegend), anti-2B4 (clone eBio244F4, Thermo Fisher Scientific), and anti-TIM-3 (clone RMT3-23, BioLegend) were used for surface staining to determine $\mathrm{T}$ cell phenotype. For the detection of cell apoptosis, splenocytes were stained with a FITC Annexin $\mathrm{V}$ apoptosis detection kit with 7-AAD (BioLegend). Tregs were identified via intracellular staining for Foxp3-APC (clone FJK-16S, eBioscience). Splenocytes were surface stained for anti-CD62L (MEL-14), antiCD69 (H1.2F3), and then permeabilized using a Foxp3 kit (BD Biosciences) and stained with anti-Foxp3, anti-CTLA-4 (UC10-489), and anti-Helios (22F6, all Abs from BioLegend). AccuCheck Counting Beads (Thermo Fisher Scientific) were added after staining to calculate the absolute number of cells per spleen.

Intracellular cytokine staining. For intracellular cytokine staining, splenocytes were stimulated with 30 $\mathrm{ng} / \mathrm{mL}$ PMA and $1 \mu \mathrm{g} / \mathrm{mL}$ ionomycin in the presence of GolgiPlug (BD Pharmingen) for 4 hours at $37^{\circ} \mathrm{C}$, and then permeabilized and fixed using BD Cytofix/Cytoperm kit followed by staining with anti-TNF (clone MP6-XT22, BioLegend) and anti-IFN- $\gamma$ (clone XMG1.2, BioLegend). Samples were analyzed on an LSRII flow cytometer (BD), and data were analyzed using FlowJo software (version 9.9.6).

Bacterial culture and cytokines in peritoneal fluid. Peritoneal fluid (PF) samples were obtained by abdominal lavage with $3 \mathrm{~mL}$ sterile saline at 36 hours after CLP. A total of $100 \mu \mathrm{L}$ PF was taken for detecting bacterial load, which were serially diluted in sterile saline and cultured on sheep's blood agar plates (Remel). Plates were incubated overnight at $37^{\circ} \mathrm{C}$ in $5 \% \mathrm{CO}_{2}$, and colony counts were determined on plates receiving $1: 10^{4}$ diluted inoculum. The rest of the PF samples were centrifuged at $4^{\circ} \mathrm{C}$ with $120 \mathrm{~g}$ for 10 minutes. Cytokine concentration was determined using the Bio-Plex 200 System according to the manufacturer's instructions (Bio-Rad). IL-6, IL-10, MCP-1 levels were reported in pg/mL. All samples were run in duplicate. Results were analyzed using Bio-Plex Manager 3.0 software.

Statistics. Data are shown as the mean \pm SEM. One-way ANOVA and multiple comparison tests were used to compare the differences among the 4 groups. Mann-Whitney $U$ tests were used to compare continuous variables between 2 groups. All statistical analyses were conducted using GraphPad Prism 8.0 software. Two-tailed $P$ values of less than 0.05 were considered significant.

Study approval. This study was conducted under approval from the Emory University IACUC (protocol 2003238-082518).

\section{Author contributions}

MLF and CMC designed the research studies. YS, TY, HS, MX, DL, and ZL conducted the experiments and the acquired data. YS and HS analyzed the data. MLF and YS wrote the manuscript. All authors edited the manuscript. 


\section{Acknowledgments}

This work was funded by NIH R01 grants AI149724, GM113228, GM104323, and GM072808 (to MLF and $\mathrm{CMC}$ ). The authors would like to thank Rafi Ahmed and Koichi Araki (Emory University) for providing LCMV.

Address correspondence to: Mandy L. Ford, 101 Woodruff Circle, Suite WMB 5105, Atlanta, Georgia 30322, USA. Phone: 404.727.2900; Email: mandy.ford@emory.edu.

1. Singer M, et al. The third international consensus definitions for sepsis and septic shock (Sepsis-3). JAMA. 2016;315(8):801-810

2. Rudd KE, et al. Global, regional, and national sepsis incidence and mortality, 1990-2017: analysis for the Global Burden of Disease study. Lancet. 2020;395(10219):200-211.

3. Hotchkiss RS, et al. Sepsis-induced immunosuppression: from cellular dysfunctions to immunotherapy. Nat Rev Immunol. 2013;13(12):862-874.

4. Watanabe E, et al. Immunoadjuvant therapy in sepsis: novel strategies for immunosuppressive sepsis coming down the pike. Acute Med Surg. 2018;5(4):309-315.

5. Boomer JS, et al. Immunosuppression in patients who die of sepsis and multiple organ failure. JAMA. 2011;306(23):2594-2605.

6. Washburn ML, et al. T cell- and monocyte-specific RNA-sequencing analysis in septic and nonseptic critically ill patients and in patients with cancer. J Immunol. 2019;203(7):1897-1908.

7. Brahmamdam P, et al. Delayed administration of anti-PD-1 antibody reverses immune dysfunction and improves survival during sepsis. J Leukoc Biol. 2010;88(2):233-240.

8. Hotchkiss RS, et al. Immune checkpoint inhibition in sepsis: a Phase $1 \mathrm{~b}$ randomized study to evaluate the safety, tolerability, pharmacokinetics, and pharmacodynamics of nivolumab. Intensive Care Med. 2019;45(10):1360-1371.

9. Chang KC, et al. Blockade of the negative co-stimulatory molecules PD-1 and CTLA-4 improves survival in primary and secondary fungal sepsis. Crit Care. 2013;17(3):R85.

10. Chen CW, et al. Cutting edge: 2B4-mediated coinhibition of $\mathrm{CD} 4^{+} \mathrm{T}$ cells underlies mortality in experimental sepsis. J Immunol. 2017;199(6):1961-1966.

11. Chen CW, et al. 2B4 but not PD-1 blockade improves mortality in septic animals with preexisting malignancy. JCI Insight. 2019;4(22):127867.

12. Yu X, et al. The surface protein TIGIT suppresses $\mathrm{T}$ cell activation by promoting the generation of mature immunoregulatory dendritic cells. Nat Immunol. 2009;10(1):48-57.

13. Johnston RJ, et al. The immunoreceptor TIGIT regulates antitumor and antiviral CD8(+) T cell effector function. Cancer Cell. 2014;26(6):923-937.

14. Chew GM, et al. TIGIT marks exhausted T cells, correlates with disease progression, and serves as a target for immune restoration in HIV and SIV infection. PLoS Pathog. 2016;12(1):1005349.

15. Kong Y, et al. T-cell immunoglobulin and ITIM domain (TIGIT) associates with CD8+ T-cell exhaustion and poor clinical outcome in AML patients. Clin Cancer Res. 2016;22(12):3057-3066

16. Beura LK, et al. Normalizing the environment recapitulates adult human immune traits in laboratory mice. Nature. 2016;532(7600):512-516.

17. Xie J, et al. Increased attrition of memory T cells during sepsis requires 2B4. JCI Insight. 2019;4(9):126030.

18. Dixon KO, et al. Functional anti-TIGIT antibodies regulate development of autoimmunity and antitumor immunity. J Immunol. 2018;200(8):3000-3007.

19. Lecoeur H, et al. Strategies for phenotyping apoptotic peripheral human lymphocytes comparing ISNT, annexin-V and 7-AAD cytofluorometric staining methods. J Immunol Methods. 1997;209(2):111-123.

20. Sebastian M, et al. Helios controls a limited subset of regulatory T cell functions. J Immunol. 2016;196(1):144-155.

21. Wing K, et al. CTLA-4 control over Foxp3+ regulatory T cell function. Science. 2008;322(5899):271-275.

22. Toomer KH, et al. Developmental progression and interrelationship of central and effector regulatory $\mathrm{T}$ cell subsets. J Immunol. 2016;196(9):3665-3676.

23. Cox MA, et al. Check point inhibitors as therapies for infectious diseases. Curr Opin Immunol. 2017;48:61-67.

24. Anderson AC, et al. Lag-3, Tim-3, and TIGIT: co-inhibitory receptors with specialized functions in immune regulation. Immunity. 2016;44(5):989-1004.

25. Joller N, et al. Cutting edge: TIGIT has T cell-intrinsic inhibitory functions. J Immunol. 2011;186(3):1338-1342.

26. Wakeley ME, et al. Check point inhibitors and their role in immunosuppression in sepsis. Crit Care Clin. 2020;36(1):69-88.

27. Kurtulus S, et al. TIGIT predominantly regulates the immune response via regulatory T cells. J Clin Invest. 2015;125(11):4053-4062.

28. Xie J, et al. Pre-existing malignancy results in increased prevalence of distinct populations of CD4+ T cells during sepsis. PLoS One. 2018;13(1):0191065.

29. Schorer M, et al. TIGIT limits immune pathology during viral infections. Nat Commun. 2020;11(1):1288.

30. Zhang Q, et al. Blockade of the checkpoint receptor TIGIT prevents NK cell exhaustion and elicits potent anti-tumor immunity. Nat Immunol. 2018;19(7):723-732.

31. Jin HS, et al. CD226(hi)CD8(+) T cells are a prerequisite for anti-TIGIT immunotherapy. Cancer Immunol Res. 2020;8(7):912-925.

32. Chauvin JM, et al. TIGIT and PD-1 impair tumor antigen-specific CD8+ T cells in melanoma patients. J Clin Invest. 2015;125(5):2046-2058.

33. Joller N, et al. Treg cells expressing the coinhibitory molecule TIGIT selectively inhibit proinflammatory Th1 and Th17 cell responses. Immunity. 2014;40(4):569-581.

34. Fuhrman CA, et al. Divergent phenotypes of human regulatory T cells expressing the receptors TIGIT and CD226. J Immunol. 
2015;195(1):145-155.

35. Bin Dhuban K, et al. Coexpression of TIGIT and FCRL3 identifies Helios+ human memory regulatory T cells. J Immunol. 2015;194(8):3687-3696.

36. Scumpia PO, et al. Increased natural $\mathrm{CD} 4+\mathrm{CD} 25+$ regulatory $\mathrm{T}$ cells and their suppressor activity do not contribute to mortality in murine polymicrobial sepsis. J Immunol. 2006;177(11):7943-7949.

37. Okeke EB, et al. $\mathrm{CD} 4+\mathrm{CD} 25+$ regulatory $\mathrm{T}$ cells attenuate lipopolysaccharide-induced systemic inflammatory responses and promotes survival in murine Escherichia coli infection. Shock. 2013;40(1):65-73. 